\title{
Study on Greenhouse Measurement and Control System Based on Zigbee Wireless Sensor Network
}

\author{
Xiaodong Zhang ${ }^{1, a}$ Ying Ding ${ }^{2, b}$ \\ ${ }^{1}$ Henan Mechanical and Electrical Vocational College, Zhengzhou, Henan, China, 451191 \\ ${ }^{2}$ Zhengzhou Institute of Technology, Zhengzhou, Henan, China, 450044 \\ aemail, bemail,
}

Keywords: Greenhouse, Measurement and Control System, Zigbee Wireless Sensor Network

\begin{abstract}
The current greenhouse control system has the problems such as the expansion is poor and the degree of intelligence is not high and so on. Based on the analysis of the characteristics of zigbee wireless sensor network, the hardware and software of the greenhouse measurement and control system are designed based on wireless sensor network. The sensor nodes and the gather nodes are designed on the hardware. Use the sensors such as temperature, the humidity, the light intensity to realize the parameter automatic gathering of greenhouse environment. The software has realized the data acquisition, the processing and the control output based on the module thought. This design has the features such as good expansion, practical, easy to operate and so on.
\end{abstract}

\section{Introduction}

In the greenhouse agricultural production process, temperature, humidity and other environmental factors directly affect the growth of crops. Therefore, environmental monitoring and control is to ensure that greenhouse production of high quality and efficiency of the important means. Conventional wired measurement and control system has the shortcomings of wiring complexity, poor anti-jamming and difficult to maintain, which restricts its popularization and application. From the 20th century, the late 90s, some European and American scientists began to study the greenhouse wireless monitoring and control system, the low-power, high reliability Zigbee technology used in greenhouse monitoring and control system, has made some experimental results. However, due to the complexity of the greenhouse environment, the wireless sensor network standards are not complete and security issues, based on Zigbee greenhouse wireless monitoring and control system development and application there are many problems to be solved.

\section{The Concept of Wireless Sensor Networks}

The wireless sensor network is a data-centric distributed multi-hop relay network composed of a large number of small nodes with integrated sensor, data processing unit and short-distance communication module. The main function is to collaboratively sense, collect and process network coverage Area, and transmits the information to the base station. Wireless sensor network system usually includes sensor nodes, gateway nodes and management nodes. A large number of sensor nodes are randomly distributed or determined to be deployed in the monitoring area, and a multi-hop wireless communication network is formed by self-organization. Sensor node is responsible for collecting the relevant information of the target, and along a path through the other nodes will be hop-by-hop data back to the convergence node, and finally by the convergence node through the Internet or satellite network data transmission to the management node.

The sensor node is usually a miniature embedded system. Sensor nodes are mainly composed of sensor module, processor module, wireless communication module and energy supply module. Sensor module is closely related to the application, including the acquisition of physical information sensors and the corresponding analog-to-digital converter (ADC). The other three modules are relatively independent of the application of the part. The wireless communication module is responsible for the information interaction with other nodes through wireless communication, such 
as receiving and sending monitoring data and exchanging control information. The energy of the wireless communication module is controlled by the wireless communication module and the wireless communication module is responsible for controlling the data. The supply of power to the module provides the energy required for the operation of the whole node, usually composed of batteries with limited energy, so the processing capacity, storage capacity and communication capacity of sensor nodes are relatively weak.

\section{The Summarize of Zigbee Agreement}

ZigBee technology name is mainly from the process of honey bees honey observation, honey bees in the process of dancing beautiful dance, the formation of ZigZag shape, in order to exchange information in order to obtain the direction of shared food source, Distance and location information. Because the honeybee itself is small, the energy required is small and the collected pollen can be transmitted, ZigBee technology is used to represent the wireless communication technology with low cost, small size, low energy consumption and low transmission rate.

ZigBee is a wireless data transmission network platform composed of 65000 wireless data transmission modules. It is very similar to the existing mobile communication CDMA network or GSM network. Each ZigBee network data transmission module is similar to a base station of the mobile network. In the entire network range, they can communicate with each other; the distance between each network node can be from the standard 75 meters to a few hundred meters after the expansion, or even a few kilometers; the other ZigBee network can also be with the existing Other various network connections. For example, a ZigBee control network in Xinjiang can be monitored over the Internet in Shanghai. The difference is that the ZigBee network is mainly for the automatic control of data transmission and the establishment of the mobile communication network is mainly for the establishment of voice communications; each mobile base station value is generally more than one million yuan, but not every ZigBee base station Each ZigBee network node can not only monitor the object itself, such as sensor nodes directly for data collection and monitoring, but also automatically transfer data from other network nodes over the data; In addition, each ZigBee network node is also Can be within the scope of signal coverage and a number of non-commitment of network information transfer task of the isolated sub-node wireless connection. Each ZigBee network node can support up to 31 sensors and controlled devices, without a sensor and controlled device terminals can have 8 different interface methods, can capture and transmit digital and analog.

\section{The Architecture of Greenhouse Measurement and Control System}

Sensor layer consists of a coordinating node, a plurality of sensing nodes and a plurality of control nodes according to the star network topology to form a wireless sensor network. The sensing layer is at the bottom of the greenhouse measurement and control system. Each node is distributed in the greenhouse according to the star topology. The environmental factors in the greenhouse are periodically collected and transmitted to the application layer through the network layer for users.

According to the role of nodes in wireless sensor networks, WSNs are divided into sensor nodes, control nodes and coordination nodes. The sensing node senses the environment object information and takes corresponding processing to send the useful information to the coordinating node. The control node receives the command information from the coordinating node, controls the switch of the equipment such as the inside and outside sunshade, the heater and so on. In addition to coordinating node's function of establishing network, it is also responsible for analyzing and summarizing the data uploaded by sensor nodes, uploading the summary information to the gateway, and coordinating nodes and transmitting the control information of Chiang gateway to the corresponding terminal nodes.

The development of sensor layer is the development of wireless sensor networks. Because of its large scale, large number of nodes and unattended, wireless sensor networks bring the challenges of cost, technology and testing for the development of wireless sensor networks. The related hardware, software and Network system development and traditional network development is very different. 
Wireless Sensor Network (WSN) is a multi-hop self-organizing network system formed by a large number of inexpensive micro-sensor nodes deployed in the monitoring area through wireless communication, which has the advantages of convenient networking and flexibility, etc. Its purpose is to collaboratively sense, and processing the information of the objects in the network coverage area and transmitting the information to the observer based on the characteristics of the wireless sensor network.

Sensor nodes collect information such as temperature, humidity, light intensity from the soil itself or the surrounding environment, complete the given monitoring task, transmit the monitoring data to the control computer for decision-making, and use the brake to control the fertilizer and moisture in the soil. Can be used to guide planting for maximum yield, and to monitor and report crop status.

Aggregation node is the intermediary between the sensor node and the management node computer in the application environment. It can connect the sensor network with the external network such as Internet or WWW, realize the protocol conversion between protocol stacks, and release the monitoring task of the management node, And the collected data forwarded to the external network.

\section{The Hardware Design and Control Theory}

The Design of Sensor Node. Sensor node is a microcomputer system specially designed for sensor networks, it is the basic unit of wireless sensor network, it is responsible for sensing and information preprocessing, in response to the instructions of the host computer to send data. It consists of five main modules: microcontroller, Radio frequency communication, non-volatile Flash memory, scalable I/O interface and power supply modules.

Microcontrollers are the core components of a sensor node that are responsible for processing data and coordinating the entire system. Typically, low-power microcontrollers with mixed-mode processing capabilities are typically available on a single chip with UART, SPI , I2C and other common interface; the other due to the need to obtain the sensor data acquisition, but also should have multi-channel analog-to-digital converter; Finally, the micro-control must have power management features, so that nodes cannot work when switching to low- ATmega128L Atmega 128L as the microcontroller unit for the following two reasons: First of all, ATmega128L RISC technology used to make it have a high computing performance; ATmega128L used in the design of high- Secondly, AT-mega128L's open-source development software tools are mature and TinyOS operating system (sensor node operating system) to support it better. Therefore, in order to more quickly build a sensor node prototype platform, select ATmega128L as the node data processing unit.

The Design of Aggregation Node. In the greenhouse measurement and control system, the sink node is a sensor network and management nodes or other external network communication bridge, in the wireless sensor network plays the role of gateway. As an ARM processor, PXA270 based on AR-MV5TE XScale architecture, Provides a powerful processing capabilities. Compared with the PXA25X series processors, PXA270 XScale micro-architecture based on the increase Intel Wireless MMX technology to improve the efficiency of multimedia processing, optimizing the processor power consumption, while adding for the mobile terminal New features in device design.

\section{Software Design and Function Analysis}

In order to make the operator can visually see all kinds of environmental parameters in the greenhouse data and distribution tasks, man-machine interface all adopt the friendly graphical interface. The system software consists of five modules: parameter setting module, data acquisition module, data analysis and processing module, control output module and data management module.

Parameter Setting Module. The administrator in the use of greenhouse monitoring and control system to use the account password to login, if the password is wrong, then prohibit access to the system due to different crops in different growth periods of the different environmental parameters, 
the same crop in different developmental stages of environmental requirements , So the environmental parameters as a control parameter to the real-time data acquisition control module to send to better meet the needs of biological growth environment. The system parameter setting function is mainly used to set the human environment requirements for the appropriate value of the system default Can be sent to the real-time data collection control module control parameters are temperature, humidity, carbon dioxide concentration, light intensity and other environmental parameters.

Data Acquisition Module. The data acquisition module mainly realizes the multichannel data synchronization acquisition, the waveform real-time display as well as the data storage function, displays the environmental factor parameter real-time change in the form of the dynamic curve or the table form. The signal acquisition task is gathers the voltage signal which the sensor gathers The acquisition channel is input to the computer through the gateway processing, and finally displayed in real time. Whether the collected signal can realize the real signal again has much to do with the choice of the sampling method and the determination of the sampling time. This system adopts the real-time sampling method.

Data Processing Module. Data processing is the process of generating information from the original data. In the system, the analog digital conversion by the A / D into the gateway, the digital display, alarm and control before, it must be carried out according to some Processing, such as: digital filtering, scale conversion, numerical calculation, logic, etc., to meet the different needs of the system.

Control Output Module. The control part of this system is mainly to realize the control of the environmental factors in the greenhouse. On the basis of the data display and alarm signal of the computer, we can operate at the interface of the actuator to meet the suitable growth environment.

Data Management Module. The data is stored in the unit of day. The collected real-time monitoring data of 24 hours is stored in the form of file, and the data file is stored in the date of data collection as the name of the file, the time of data collection and Data as the file storage content, so you can easily meet the operation of the file. At the same time can be analyzed, statistics and printing.

\section{Conclusion}

With the development of facility horticulture in China in recent years, the greenhouse control system and expert system have played an important role in greenhouse development. Greenhouse environment control system can automatically detection and display the greenhouse interior and exterior environment; according to the requirements of different crops, make multi-factor comprehensive adjustment and control; at the same time it can store the data for a long time for the greenhouse environmental factors to meet the needs of scientific research and production, accumulated a wealth of information data for the intelligent agriculture expert system.

\section{References}

[1] Li Jingyuan. Microelectronics and Computer Science, Vol. 6 (2014) No 53, p.25-26

[2] Peng Sue, Wang Yunhui, Wang Qunyong. Sensor Technology, Vol. 12 (2015) No 27, p.74-76

[3] Qian Xiyuan, Jing Jianfen. Mechatronics, Vol. 30 (2014) No 19, p.144-145

[4] Wang Kuailiang. Measurement and Control Technology, Vol. 29 (2008) No 27, p.21-23

[5] Zhang Gongxu, Sun Jing. Computer Engineering and Design, Vol. 8 (2013) No 27, p.57-60 